\title{
A new method for assessing motion-in-depth perception in strabismic patients
}

\author{
Y Watanabe, ${ }^{1}$ T Kezuka, ${ }^{1}$ K Harasawa, ${ }^{1}$ M Usui, ${ }^{1}$ H Yaguchi, ${ }^{2}$ S Shioiri ${ }^{3}$
}

\begin{abstract}
${ }^{1}$ Department of Ophthalmology, Tokyo Medical University Hospital, Tokyo, Japan; ${ }^{2}$ Department of Information and Image Sciences Chiba University, Japan; ${ }^{3}$ Research Institute of Electrical Communication Tohoku University, Japan
\end{abstract}

Correspondence to: Y Watanabe, Department of Ophthalmology, Tokyo Medical University Hospital, 6-7-1 Nishishinjuku, Shinjuku-ku, Tokyo 160-0023, Japan; maxi_xyz@yahoo.co.jp

Accepted 14 June 2007 Published Online First 27 June 2007

\section{ABSTRACT}

Aim: In strabismus clinics, stereoscopic depth perception is usually examined using static stimuli, but these stimuli do not necessarily allow assessment of the ability to perceive motion in depth. We assessed the ability to perceive motion-in-depth perception using a novel stereo motion test that we developed and compared with that to perceive static depth perception using a conventional stereo test in strasbismic patients.

Methods: To investigate motion-in-depth perception in patients with strabismus, we developed a stereo motion test using four types of computer-generated dynamic visual stimuli. Three of them are random dot stereograms of two parallel planes moving in depth. The patient is asked to indicate the planes' direction of rotation in depth (in the first and second types) or the presence/absence of motion-in-depth signal (in the third type). The fourth type of stimulus was a random dot stereogram of a rotating cylinder. The upper and lower parts of the cylinder rotate in opposite directions, and the patient is asked to indicate the position of the border between the two parts.

Threshold disparity was defined as the disparity (relative disparity between the nearest and farthest points of the planes or the cylinder) that gives a critical level of performance with the method of limit. The conventional Titmus stereo test using static visual stimuli was used to assess static depth perception. The measurements were performed in 52 strabismic patients, aged between 4 and 38 years old, who visited Tokyo Medical University Hospital between January 2003 and July 2004.

Results: The results showed a poor correlation in the threshold of individual patients between the stereo motion test and conventional Titmus stereo test. For example, the ability to perceive motion in depth (disparity threshold $<500 \mathrm{sec}$ of arc) was demonstrated in three of seven patients who were not able to perceive depth using static stimuli (0/9 for Titmus circle). These results suggest that the process of the dynamic element of binocular depth perception is preserved in some of the strabismic patients who lack static stereopsis.

Conclusion: This study indicates the importance of testing motion-in-depth perception as well as static depth perception in assessing stereopsis in strabismic patients.

The measurement of stereopsis is an important component of ocular assessment or vision screening procedure. In strabismus clinics, the Titmus stereo test which uses static visual stimuli is often used for the measurement of stereopsis. In everyday life, however, we often see moving objects. Depth perception is influenced considerably by motion, and motion is an important cue while catching a ball, driving a car, avoiding collision with somebody or watching a three-dimensional (3D) motion picture in the recent world with new technologies. ${ }^{1-3}$ For evaluating the ability of stereopsis in these situations, we should assess the motion-in-depth perception. Although the Titmus stereo test is usually used to examine stereopsis, the test is limited to static depth perception and cannot be applied to the dynamic aspects of depth perception, such as perception of motion in depth. It is necessary to consider the fact that motion-in-depth cues are processed in pathways different from those for static depth cues. The human retino-geniculate-cortical visual system is divided into at least two pathways. ${ }^{4}$ Hammarrenger et $a l^{5}$ investigated whether the magnocellular and parvocellular pathways mature at the same rate or follow different developmental courses. Pattern visual evoked potentials (PVEPs) were recorded in 33 infants aged between 0 and 52 weeks, in response to two spatial frequencies ( 0.5 and $2.5 \mathrm{c} / \mathrm{deg})$ presented at four contrast levels (4, 12, 28 and 95\%). They concluded that the two principal visual pathways appear to follow different maturation course and that the magnocellular pathway functions earlier and matures faster than the parvocellular pathway during the first year of life. Hatch and Laudon ${ }^{6}$ reported on the long-term follow-up of a patient who had constant esotropia from around age 3 years 4 months to age 9 years 7 months. After orthoptic treatment at age 9 , he subsequently developed bifoveal fixation as measured by $30 \mathrm{sec}$ arc of contour stereopsis and 250$500 \mathrm{sec}$ arc of random dot stereopsis. This case demonstrates that bifoveal fixation can be obtained after longstanding strabismus, and suggests that the human visual system adapts flexibly to the environment is related to the sensitive period of stereopsis. ${ }^{7}$ As mentioned above, assessment of dynamic stereopsis is important because our retinal images are constantly in motion, due to the motion of oneself or of the objects in the environment in everyday life. A recent study reported that motion in depth was perceived in about half of the esotropic patients who do not have fine stereopsis. ${ }^{8}$ From the scientific point of view, there are at least two possible binocular cues for seeing motion in depth ${ }^{10}$ (fig 1). One is binocular disparities at different times, and the other is interocular velocity differences. There are many reports on stereopsis in esotropia, but only a few reports on motion in depth in exotropia.

In this study, we developed a new computergenerated dynamic stereo test using four types of dynamic visual stimuli. Disparity and motion cues can be included or separated independently in this program. The purpose of this study was to determine the characteristics of esotropic and exotropic patients who have good motion-in-depth 
Figure 1 (a) Diagram of the retinal images when an object moves in depth. There are two possible cues to see motion in depth: (A) disparity with change in time and $(B)$ interocular velocity differences. (b) The kinematograms contained two frames for each eye, and the displacement of the left image was in the opposite directions to that of right image. There was no correlation between the left and right image.

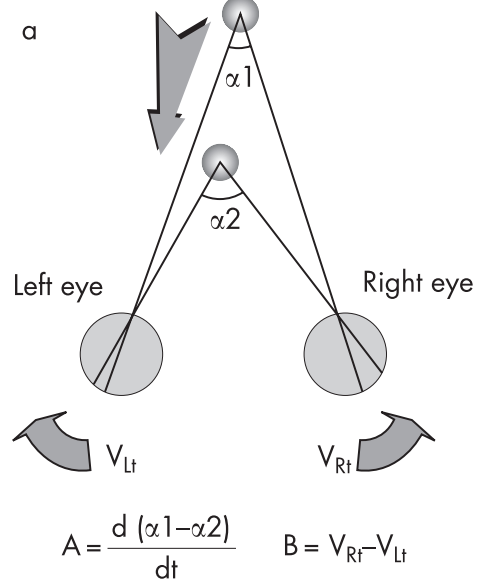

b Left stimulus

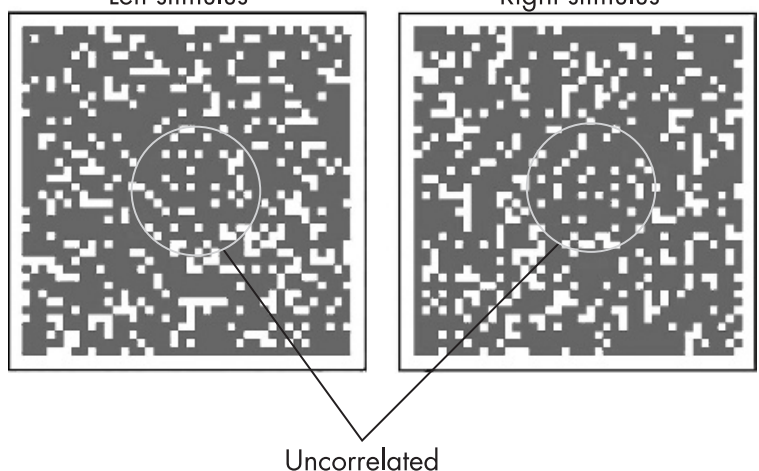

Uncorrelated
号

¿ perception but poor static stereopsis, by measuring the threshold disparity with a notebook computer.

\section{MATERIALS AND METHOD}

\section{Strabismic patients}

The study was approved by the ethics committees of the Tokyo Medical University. The research followed the tenets of the Declaration of Helsinki, and informed consent was obtained from all adult subjects and parents of participating children after the nature and possible consequences of the study were explained. This study was performed at the Department of Ophthalmology of Tokyo Medical University between January 2003 and July 2004. The measurement was conducted in 52 strabismic patients (32 female and 20 male) with ages ranging from 4 to 38 years (mean 16 years). Thirty-three exotropia patients and 19 esotropia patients were studied. Patients with vertical deviation are excluded. All patients visited consecutively. Patients with a medical history of ophthalmic operation (including strabismus operation and others) were excluded. As controls, we examined six normal subjects (four men and two women; age 22-30 years; all subjects without any strabismus) without any ocular abnormality other than refractive errors. Ophthalmic examinations including visual acuity and the Titmus stereo test were performed on all patients. The angle of near $(30 \mathrm{~cm})$ and far $(5 \mathrm{~m})$ strabismus was measured by the simultaneous prism cover test, as were exotropia and exophoria. Exclusion criteria were esotropia from organic disorders, premature birth (gestation before 37 weeks) and delayed development.

\section{Visual stimulator and procedure}

Stimuli were displayed on a 15" colour monitor (Sharp PCMV1-VC1, Tokyo) on a notebook computer, programmed by MATLAB with Psychophysics Toolbox. ${ }^{11}$ Anaglyphic randomdot stereograms were used. The stimulus size was $340 \times 340$ pixels, corresponding to $9.4 \times 9.4$ degrees at a viewing distance of $50 \mathrm{~cm}$. Four tests were conducted with different types of stimuli (fig 2). In test 1 , the stimuli were random dot stereograms of two parallel planes moving in depth (rotating about vertical axis either clockwise or counterclockwise). The stimuli contained cues of both binocular disparities at different times and interocular velocity differences. In test 2 , the stimuli were the binocularly uncorrelated version of the random dot patterns used in test 1 , such that the stimuli did not contain the disparity cues. In this stimulation (tests 1 and 2), the patient as well as the normal subjects saw the random-dot pattern rotated in depth about the vertical axis without changing the surface orientation (always facing to the observer). In other words, all the dots moved in the same way on the screen to generate the same path in the $3 \mathrm{D}$ space. In test 3 , we presented two random dot stereograms. The stimuli were dynamic random dot stereograms, which were the temporally uncorrelated version of the random dot patterns used in test 1 , such that the stimuli did not contain the velocity cues. The motion in this case was oscillation in depth instead of rotation (fig 2). The patients were asked to report whether or not the figure appeared to move back and forth in a "Z" direction. In test 4, the stimuli random dot stereograms represent a rotating cylinder. The upper and lower parts of the cylinder rotated in opposite directions. Patients were asked to describe the position of the border between the two parts. To detect the border, both disparity and velocity cues are necessary. At the beginning of each trial, the computer gave a start signal (a long beep sound), and then the visual stimulus appeared and started to move. After the presentation duration of $3 \mathrm{~s}$, the patients were asked to indicate the direction of rotation in depth in tests 1 and 2 , select the figure that appeared to move in depth in test 3 or locate the border in test 4 . We used the method of limit to measure the threshold, changing the disparity between 1200 and $100 \mathrm{~s}$. With the decrease in disparity, motions along the depth axis become smaller, while the horizontal axis remain constant. The orbit of the plane rotation changed from circular to ellipse while decreasing the disparity.

\section{Statistical analysis}

The correlation coefficient was used to analyse the results of the Titmus stereo test and motion-in-depth perception. An
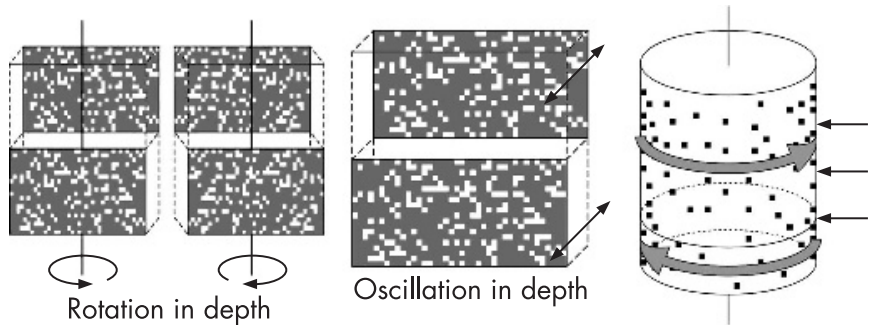

Figure 2 Stimuli used in the novel stereo motion test. Left figure shows how two planes move in tests 1 (two cues condition) and 2 (velocity cue condition). Centre figure shows how two planes move in test 3 (disparity cue condition). Right figure shows the rotating cylinder used in test 4. The upper and lower parts of the cylinder rotate in opposite directions. 
Figure 3 (A) Correlation of disparity threshold between the Titmus stereo test and stereo motion test (test 1). Each symbol represents disparity thresholds of the two tests of each patient. The unit of both axes is sec of arc. (B) Correlation of disparity threshold between velocity cue and disparity cue. Each symbol represents disparity thresholds of the two tests of each patient. The unit of both axes is sec of arc. The black symbol is a normal subject (all of the normal subjects have normal stereopsis; Titmus circles 9 / 9).
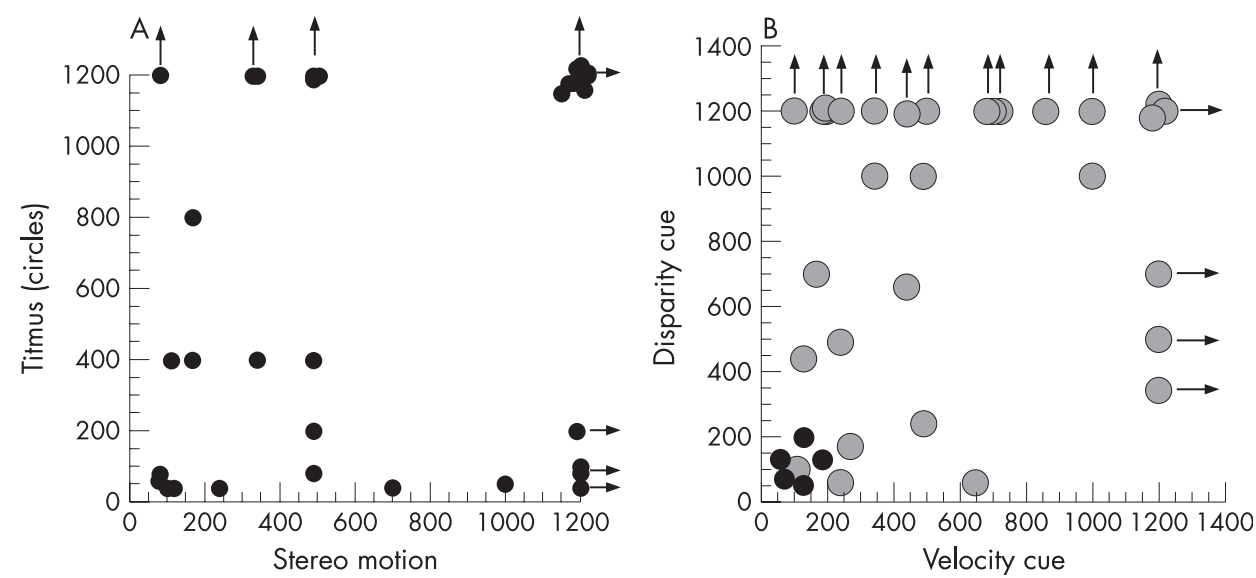

unpaired t test was used to assess the relation between ocular alignment and the measurement of each stereo test (Titmus circle or one of four stereo motion tests). A chi-square test was used to evaluate the difference of achievement in the Titmus stereo test among different groups of strabismus patients. All $p$ values less than 0.05 were accepted as statistically significant.

\section{RESULTS}

Figure 3 shows the correlation in disparity threshold between test 1 (stereo motion test) and the Titmus stereo test and that between test 2 (velocity cue test) and test 3 (disparity cue test). This figure shows the correlation in disparity threshold between test 1 (stereo motion test) and the Titmus stereo test and that between test 2 (velocity cue test) and test 3 (disparity cue test). The threshold of the velocity cue test is actually the amplitude of lateral motion, but we use the equivalent disparity value to compare the results with other conditions. The equivalent disparity is the disparity of the stereo motion test from which the binocular correlation is removed to make the velocity cue test. Although the thresholds in some patients in either/both tests were too high to measure (data with arrows data at the highest disparity in the figures indicate that the patients were not able to perceive depth at the maximum disparity used), static and/or dynamic disparity thresholds could be measured in most of the patients. This figure shows that some patients who cannot see static depth can see motion in depth. On the other hand, some patients who can perceive static depth cannot see motion in depth. For example, among seven patients who could not perceive depth using the largest depth stimulus in Titmus stereo test (data points at $1200 \mathrm{sec}$ of arc of horizontal axis, or the right side of the panel), four patients could see motion in depth at a threshold of $500 \mathrm{sec}$ of arc or smaller. The correlation coefficient of disparity threshold between the stereo motion test (test 1) and Titmus stereo test is as low as 0.094 . The patients were divided by ocular alignment at a cut-off of 20 prism dioptres and analysed statistically by t test, and $p<0.05$ was accepted as statistically significant. There was no statistical difference between alignment within 20 prism dioptres and more than 20 prism dioptres in the esotropic patients $(p=0.45$ by t test) (table 1). On the other hand, there was a significant difference between alignment within 20 prism dioptres and more than 20 prism dioptres in the exotropic patients ( $p=0.046$ by t test) (table 1).

\section{DISCUSSION}

In this study, we examined the ability to see motion-in-depth in esotropic and exotropic patients. The patient who can see motion in depth in test 2 should be considered, as they make use of velocity differences between the left and right retinal images, while those who can see motion in depth in test 3 should be considered, as they make use of disparity change in time. ${ }^{10}$ In this study, we demonstrated that motion-in-depth perception was present in more than $25 \%$ of the strabismic patients who do not have fine stereopsis. Fawcett et $a l^{12}$ showed that postsurgical correction of orthophoria supports better stereoacuity than a larger residual angle of strabismus subtending up to 8 prism dioptres of deviation. However, in dynamic condition, ocular alignment within 10-15 prism dioptres is an important factor in obtaining binocular motion in depth perception. ${ }^{8}$ From the above findings, recovery of stereoscopic vision may be possible in a larger number of cases than was presumed previously. Our results show that ocular alignment within 20 prism dioptres is important to achieve stereo motion perception. Thus, plasticity of the velocity motion condition is greater than that of the static disparity condition. Recovery of stereoscopic vision by strabismus surgery may be possible in a larger number of cases than was presumed previously. Our result suggests that the process to perceive static depth is different from the process to

Table 1 Interaction between stereo test and ocular alignment

\begin{tabular}{|c|c|c|c|c|c|c|}
\hline & \multicolumn{4}{|c|}{ Stereo motion test } & \multicolumn{2}{|c|}{ Titmus stereo } \\
\hline & Two cue & Velocity cue & Disparity cue & Cylinder cue & Fly & Circles \\
\hline Esodaviation & ns & 0.046 & ns & ns & $\mathrm{ns}^{*}$ & ns \\
\hline Exotropia & 0.043 & 0.043 & ns & ns & $\mathrm{ns}^{*}$ & ns \\
\hline
\end{tabular}

Patients were divided into two groups of ocular alignment within 20 PD and ocular alignment over 20 PD. The unpaired t test was used to assess the relation between ocular alignment and different elements of the stereo-motion tests.

${ }^{*}$ Chi-square test was used for comparison between the Fly in the Titmus stereo test and ocular alignment. Figures are p values of significant tests.

ns, not significant. 
Table 2 Correlation coefficient of disparity threshold between each pair of tests

\begin{tabular}{lcccc}
\hline & Stereo motion (test 1) & Velocity cue (test 2) & Disparity cue (test 3) & Rotating cylinder (test 4) Titmus stereo test (circles) \\
\hline Stereo motion (test 1) & & & & \\
Velocity cue (test 2) & 0.71 & & \\
Disparity cue (test 3) & 0.184 & 0.311 & -0.04 & \\
Rotating cylinder (test 4) & 0.016 & -0.002 & 0.11 & 0.26 \\
Titmus stereo test (circles) & -0.09 & -0.1 & \\
\hline
\end{tabular}

perceive dynamic depth. This is confirmed by the low correlation of the thresholds between the Titmus stereo test and test 2, 3 or 4 (table 2). The mechanism responsible for the detection of static depth seems to play no important role for the detection of dynamic depth. It is likely, therefore, that the process of the dynamic element of binocular depth perception is preserved in some of the strabismic patients who lack static stereopsis. ${ }^{13}$ The high correlation (0.71) of thresholds between tests 1 and 2 suggests that velocity information is important to see motion in depth. In this study, we investigated motion in depth in strabismic patients using four different types of stimulus. The results suggest that all of the tests can be used to assess the dynamic elements of binocular stereopsis. Test 1 may be the first choice for testing dynamic stereopsis because it uses common stereo motion stimuli with binocular cues. Tests 2, 3 and 4 , however, perhaps provide more detailed information of the problem of each patient. The low correlation between tests 2 and 3 (velocity cue and disparity cue conditions) suggests that the process for disparity cue and the process for velocity cue are different, as has been suggested previously. ${ }^{10}$ The two tests can be used to examine different aspects of motion in depth perception. Stereo vision examination is important as a guide to preserve postoperative simultaneous perception as well as assessment of binocular functions of the patient. Examination of stereo vision using dynamic stimulation as well as assessing static fine stereopsis may be useful for these purposes.

\section{CONCLUSION}

We developed a method to assess the ability to perceive motion in depth, or dynamic stereopsis, in patients with strabismus. Using the novel method, we found that some (four of seven) patients with strabismus who do not have fine stereopsis were able to detect motion in depth. For appropriate assessment of the patient's ability of binocular vision, assessments using dynamic stimuli such as those that we have developed are important.

Competing interests: None declared.

\section{REFERENCES}

1. Lee S, Shioiri S, Yaguchi H. Effects of temporal frequency and contrast on spatial frequency characteristics for disparity threshold. Opt Rev 2003;10:120-3.

2. Lee S, Shioiri S, Yaguchi $H$. The effect of exposure duration on stereopsis and its dependency on spatial frequency. Opt Rev 2004;11:256-264.

3. Shioiri S, Morinaga A, Yaguchi H. Binocular depth perception for moving objects. In: Javidi B, Okano F, eds. 3D television, video and display technology, Berlin: Springer, 2002:397-427.

4. Perry AH, Oehler R, Cowey A. Retinal ganglion cells that project to the dorsal lateral geniculate nucleus in the macaque monkey. Neuroscience 1984;12:1101-23.

5. Hammarrenger $\mathbf{B}$, Lepore F, Lippe $\mathbf{S}$, et al. Magnocellular and parvocellular developmental course in infants during the first year of life. Doc Ophthalmol 2003;107:225-33.

6. Hatch SW, Laudon R. Sensitive period in stereopsis: random dot stereopsis after long-standing strabismus. Optom Vis Sci 1993;70:1061-4.

7. Fawcett SL, Wang $Y Z$, Birch EE. The critical period for susceptibility of human stereopsis. Invest Ophthalmol Vis Sci 2005;46:521-5.

8. Maeda M, Sato M, Ohmura T. Binocular depth-from-motion in infantile and lateonset esotropia patients with poor stereopsis. Invest Opththalmol Vis Sci 1999;40:3031-6.

9. Cumming BJ, Perker AJ. Binocular mechanisms for detecting motion-in-depth. Vision Res 1994;34:483-95.

10. Shioiri S, Saisho H, Yaguchi H. Motion in depth based on inter-ocular velocity differences Vision Res 2000:40:2565-72.

11. Brainard DH. The psychophysics toolbox. Spatial Vision 1997;10:433-6.

12. Fawcett SL, Stager DR Sr, Felius J. Factors influencing stereoacuity outcomes in adults with acquired strabismus Am J Ophthalmol 2004;138:931-5.

13. Fujikado T, Hosohata J, Ohmi G, et al. Use of dynamic and colored stereogram to measure stereopsis in strabismic patients. Jpn J Ophthalmol 1998:42:101-7. 